\title{
Graphene transfer process and optimization of graphene coverage
}

\author{
Syarifah Norfaezah Sabki ${ }^{1}$, Shafiq Hafly Shamsuri ${ }^{1}$, Siti Fazlina Fauzi ${ }^{1}$, Meghashama Lim Chon-Ki ${ }^{1}$ and Noraini Othman $^{1}$ \\ School of Microelectronic Engineering, Universiti Malaysia Perlis (UniMAP), Kampus UniMAP Pauh Putra, 02600 Arau, Perlis, \\ Malaysia.
}

\begin{abstract}
Graphene grown on transition metal is known to be high in quality due to its controlled amount of defects and potentially used for many electronic applications. The transfer process of graphene grown on transition metal to a new substrate requires optimization in order to ensure that high graphene coverage can be obtained. In this work, an improvement in the graphene transfer process is performed from graphene grown on copper foil. It has been observed that the graphene coverage is affected by the pressure given to the top of PDMS to eliminate water and air between graphene and $\mathrm{SiO}_{2}$ (new substrate). This work experimented with different approaches to optimize the graphene coverage, and stamping method has proven to be the best technique in obtaining the largest graphene coverage. This work also highlights the elimination of impurities from graphene after the transfer process, known to be PMMA residues, which involved immersion of graphene in acetone. This method has improved the graphene conductivity.
\end{abstract}

\section{Introduction}

Since its first discovery by the two physicists from Manchester University in 2004 [1], graphene has become a trending in research and applications [2-5]. In recent years, many applications have been explored using graphene as the base material due to its remarkable characteristics, particularly mechanical and electrical properties. This makes graphene very practical and feasible to be incorporated in electronics and RF applications [6-11]. In order to incorporate graphene in devices for applications, the right transfer process plays a major role in order to produce well function devices.

Graphene grown on transition metals have been incorporated in many electronic applications due to its quality and coverage of monolayer graphene. The transfer method of this graphene on transition metal is important in order to ensure a high coverage of graphene is transferred and graphene quality is sustained.

There is several graphene transfer methods have been carried out in graphene researches. One of the method is using a polymeric layer which are polymer supported transfer (PST) [12]. Another well known transfer method is known as direct transfer [13] and pick up transfer [14]. All the three methods use polymethil methacrylic (PMMA) as the support layer for graphene. So far, these three methods have successfuly transferred graphene on to substrates $[12,13,14]$. Among all the methods, the graphene transfer using polymeric layer have been popular due to its easy manage and better yield. However graphene transferred by using this method suffers crack and impurities which is undesirable for a device. Therefore this paper discusses about a new approach that can be applied to optimize the graphene coverage and eliminate impurities from the graphene layer after it is transferred to a new substrate surface.

\section{Methodology}

\subsection{Transfer process}

In the graphene transfer, the process started with spin coating the graphene on copper $(\mathrm{Cu})$ surface with polymethil methacrylate (PMMA), followed with drops of polymethyl siloxane (PDMS). Both polymers are cured at $\sim 100^{\circ} \mathrm{C}$ to solidize and harden. The following step is to etch the $\mathrm{Cu}$ foils in aqueous iron chloride $\left(\mathrm{FeCl}_{3}(\mathrm{aq})\right)$, leaving graphene attached to PMMA and PDMS. PMMA is used because this polymer can be easily dissolved in acetone. PDMS works as a protective layer and it eases the sample handling.

After the $\mathrm{Cu}$ etching process, the graphene on PMMA/PDMS is cleaned in distilled water and left on the water surface to be scooped using a spatula, and finally placed on a targeted substrate. In this case of study, silicon dioxide $\left(\mathrm{SiO}_{2}\right)$ is used as the substrate. Three graphene on copper samples are transferred based on this method. The process followed with giving a significant pressure to the top of the PDMS, which we believe affects the coverage of graphene that will be obtained on $\mathrm{SiO}_{2}$. Applying pressure to the top of the PDMS is important to eliminate excess water and air because during the transfer, water and air is trapped between both medium, which could lead to non-uniform coverage of graphene.

To optimize the graphene coverage, three different approaches in applying pressures to the top of PDMS are performed. The approaches are illustrated in Fig. 1. The first approach (Fig. 1(a)) is performed by giving pressure to the top of PDMS using finger tip and the pressure is given repeatedly in $10 \mathrm{~s}$. This sample is marked as sample 1. The second approach (Fig. 1(b)) is performed by applying a constant pressure for $\sim 10 \mathrm{~s}$ using fingertip and the sample is marked as sample 2 . The final step is performed by applying a constant

\footnotetext{
*Syarifah Norfaezah Sabki: faiesns@gmail.com
} 
pressure (also at $\sim 10$ s) using a wooden block with very flat surface and similar surface area as the graphene area (Fig. 1(c)), marked as sample 3.

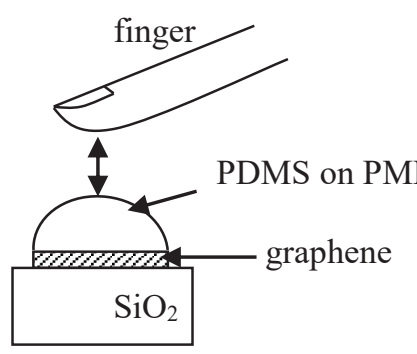

(a)

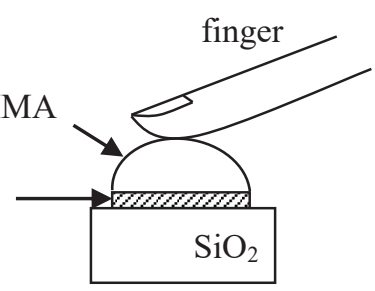

(b)

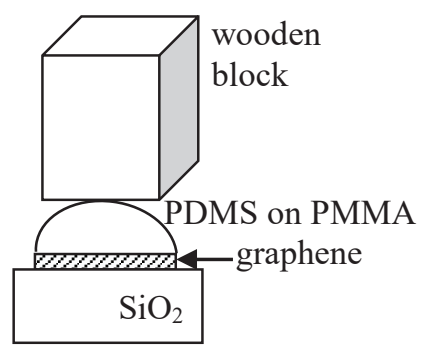

(c)

Fig. 1. Approaches taken to eliminate water and air between graphene and $\mathrm{SiO}_{2}$ to optimize the graphene coverage (a) finger tip is pressed repeatedly in 10 s (sample 1), (b) finger tip is pressed constantly for 10s (sample 2), (c) wooden block with flat surface is pressed constantly for 10 s (sample 3 ).

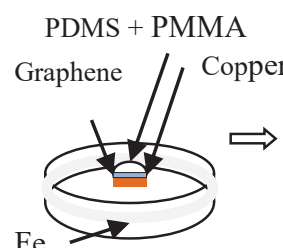

(a)

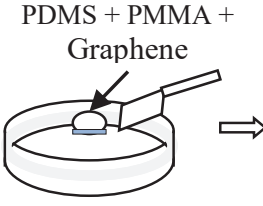

(b)

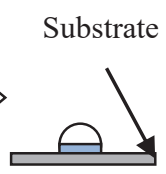

(c)
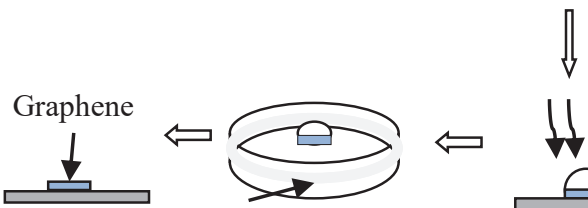

(f)

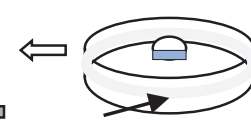

(e)

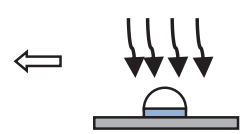

(d)
Fig. 2. Transfer process. (a) PDMS + PMMA + graphene $+\mathrm{Cu}$ are etched in $\mathrm{FeCl}_{3}$. (b) PDMS + PMMA + graphene are transferred onto substrate using spatula. (c) PDMS + PMMA + graphene are being pressed down onto new substrate $\left(\mathrm{SiO}_{2}\right)$ surface. (d) The sample is baked in convection oven. (e) PDMS + PMMA + graphene are immersed in acetone. (e) PMMA is dissolved, leaving graphene on $\mathrm{SiO}_{2}$.

After all the three different approaches are performed, all the samples are then annealed in a furnace at $\sim 100^{\circ} \mathrm{C}$ for $\sim 10$ minutes to ensure the samples are completely dry and van der Waals force between graphene and $\mathrm{SiO}_{2}$ surface is formed. The samples are then immersed in acetone to dissolve the PMMA, leaving graphene attached on $\mathrm{SiO}_{2}$. The transfer process is illustrated in Fig. 2.

\subsection{Characterization of the transferred graphene layer.}

The surface characteristics of the graphene layer are observed using atomic-force microscopy (AFM), SPA400 from SII Instrument. AFM images are taken by using tapping mode with the scale of $5 \times 5 \mu \mathrm{m}$ and scan speed of $1 \mathrm{~Hz}$ to obtain high resolution topographic images. The image of the transferred graphene layer on $\mathrm{SiO} 2$ is captured by using High Power Microscope (HPM) ECLIPSE L300N from Nikon. The magnification of $5 \times 0.15 \mathrm{~A}$ is used to inspect the transferred graphene layers quality and coverage. For further investigation, the electrical test on the transferred graphene layers is carried out by using two points probe, Semiconductor Parameter Analyser (SPA) 4200-SCS from KEITHLEY. The electrical test result is analysed by using KITE software with the voltage sweep $-10 \mathrm{~V}$ to $+10 \mathrm{~V}$ and step of 0.5 .

\section{Results and Discussions}

\subsection{Surface characteristics of the transfered graphene}

As shown in Fig. 1, three transferred graphene samples (Sample 1, 2, and 3) are investigated to observe of which sample produced the best graphene quality after the transfer process. Based on sample 1 which is pressed with finger tip repeatedly produced graphene patches that are not uniform across the substrate surface as shown in Fig. 3(a). The repeated pressure given to the top of PDMS cause disturbance between graphene and the substrate surface, which lead to high degree of graphene edge defects and non-uniform graphene coverage. This result is undesirable for electronic applications, where a more controlled surface coverage is required.

Therefore, sample 2 is prepared by pressing finger tip on the top of PDMS firmly and constantly for about ten seconds to ensure that the graphene is well attached to the substrate surface. The optical microscopy image in Fig. 3(b) shows that there is a larger coverage of graphene compared to sample 1. However a small amount of graphene patches are still observed. 


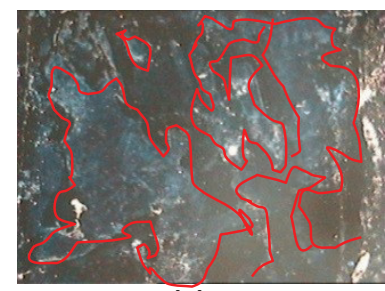

(a)

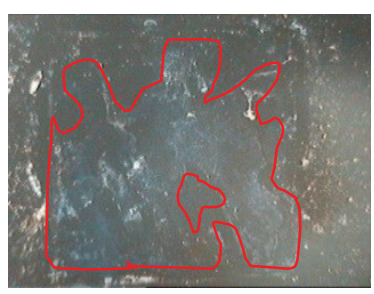

(b)

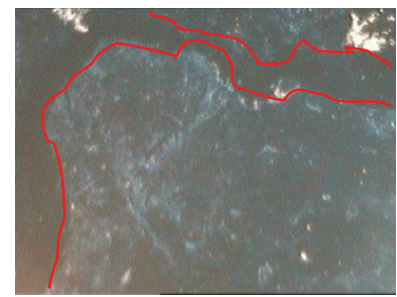

(c)

Fig.3. Optical microscopy image of graphene on $\mathrm{SiO}_{2}$ after transfer process. The size of the image is equivalent to the size of actual area of the transferred graphene, $1 \mathrm{~cm} \times 1 \mathrm{~cm}$. (a) Sample 1- finger tip is pressed repeatedly in 10s, (b) Sample 2 - finger tip is pressed constantly for 10s, (c) Sample 3 wooden block with flat surface is pressed constantly for $10 \mathrm{~s}$ (Stamping method).

Graphene on Sample 3 is transferred by pressing the top of PDMS using a wooden block that has a flat surface with dimension of $1 \mathrm{~cm} \times 1 \mathrm{~cm}$, which is exactly similar to the size of the overall graphene sample. The optical microscopy image of the graphene after the transfer process as in Fig. 3(c) shows that a large coverage of graphene is obtained. The graphene coverage in sample 3 is the largest compared to sample 1 and 2 . This shows that, by pressing the graphene sample with a flat wooden block of the same size as the graphene sample yielded the best graphene coverage. Since the action of pressing a wooden block is similar to stamping process, therefore we named this process as 'stamping method'. Note that we have tried different sizes of wooden block and we observed that smaller surface area of the wooden block reduced the graphene coverage. Hence a larger surface area of the tool is required because a more stable pressure can be applied.

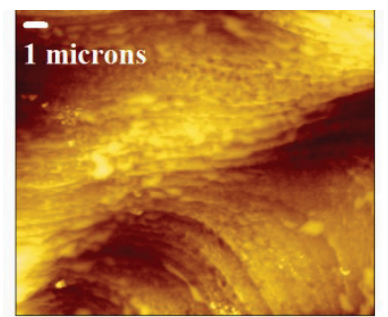

(a)

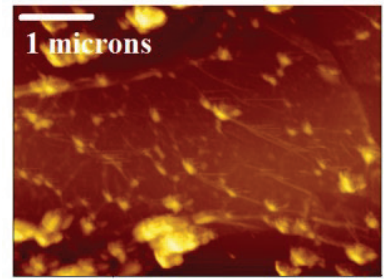

(b)
Fig.4. AFM images of (a) untreated graphene on copper foil before transfer process (b) graphene transferred to $\mathrm{SiO}_{2}$ (Sample 3 - by stamping method using wooden block), immersed 1 time in acetone for $30 \mathrm{~min}$.
Fig. 4(a) above shows graphene on $\mathrm{Cu}$ foil of thickness $0.25 \mu \mathrm{m}$, with copper orientation in (111). The step edges of $\mathrm{Cu}$ can be seen clearly. After the transfer process, the $\mathrm{Cu}$ step edges is no longer present, however graphene cracks can be seen as in Fig. 4(b). It is also obvious that there are impurities on the graphene layer, as represented by the spots with lower contrast. Possible impurities are dusts or polymer residues that are also transferred during the transfer process. However, it is suggested that dusts are already removed with only 1 time immersion in acetone, because only van der Waals force is present. To remove polymer residues requires more immersion time or duration in acetone.

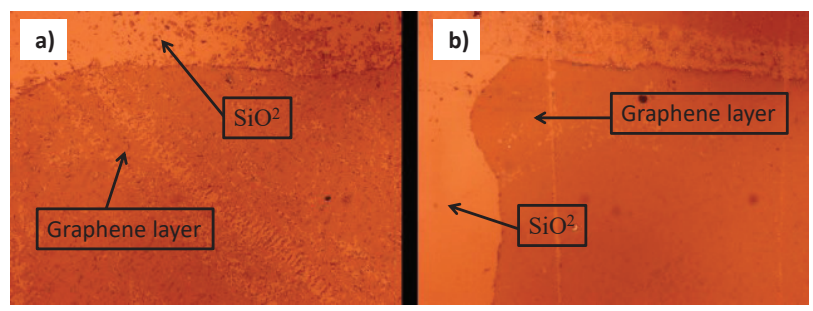

Fig.5. Optical microscopy images of transferred graphene layer on $\mathrm{SiO}^{2}$ (a) 1 time immersion in acetone for $30 \mathrm{~min}$ and (b) 3 times immersion in acetone with immersion of 30 min each time.

Not only having outstanding electrical properties, graphene is also well known by its optical properties which can be used as high transparent electrode in nanoelectronic devices. The transparency of the graphene layer can be seen in Fig. 5, where Fig.5 (a) shows that graphene layer that is transferred in this work is slightly high in contrast, which may be suggested by the presence of impurities. The possible residues may be suggested by PMMA that is used in the transfer process, where it may not be totally dissolved. There should be no PDMS residues on the graphene surface because PDMS does not dissolve in acetone and it is not attached directly on graphene. Dissolving PMMA in acetone is one typical technique that is easy to conduct, low cost and feasible. Another method that can be used is to anneal the sample with PMMA in ultra high vacuum (UHV) system, which will evaporate and remove the PMMA physically [15]. However the process requires high cost, complicated system and difficult to conduct.

As shown in Fig. 5(b), after further cleaning, better surface area is obtained, in which the transparency is improved. The image of graphene on $\mathrm{SiO}_{2}$ in Fig. 5(b) shows that graphene layer is more apparent. Such sample surface is obtained after 3 times immersion in acetone, with each immersion; the sample is left in acetone for about 30 s. It is also suggested that the immersion of the sample in acetone can be done longer than 2 hours to obtain the same result.

It is important to ensure all the polymer residues are eliminated from the graphene after the transfer process to reduce the effect of graphene defects and to optimize the graphene quality. High amount of PMMA residues may affect the performance of graphene layer for any electronic application, since PMMA is non 
conductive. It has been investigated that polymer residue such as PMMA provides scattering sites that may alter the electronic structure of graphene especially its mean free paths of carrier charges [16].

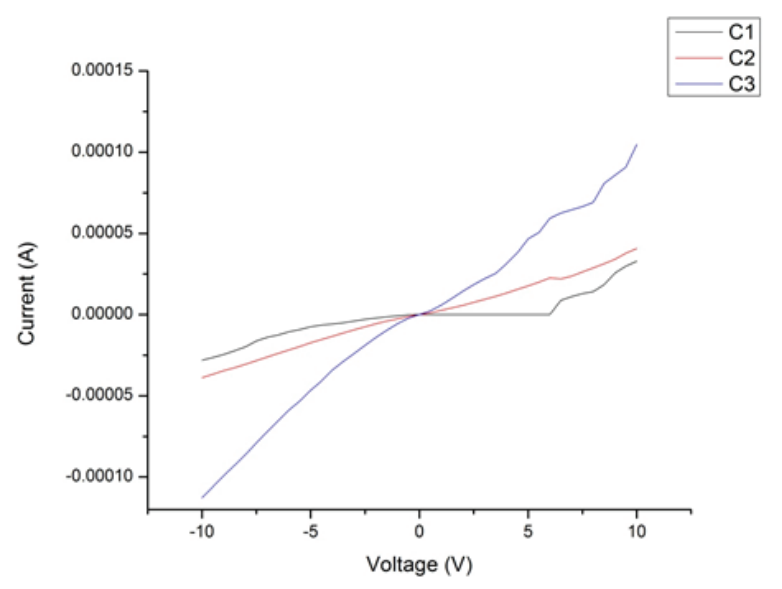

Fig.6. I-V curve of transferred graphene layer obtained from sample $1(\mathrm{C} 1)$, sample $2(\mathrm{C} 2)$ and sample $3(\mathrm{C} 3)$.

To observe the quality of the obtained graphene layer after the transfer process, IV measurement is performed. The IV characteristics in Fig. $\mathbf{6}$ is referred to sample 3 (stamping method using wooden block) that has undergone 3 times immersion in acetone $(30 \mathrm{~min}$ each immersion). The result of the first immersion is represented by curve $\mathrm{C} 1$, followed with second immersion represented by curve $\mathrm{C} 2$ and the third immersion is represented by curve $\mathrm{C} 3$. It is observed that $\mathrm{C} 1$ shows the lowest conductivity, and C3 shows the highest conductivity. Even though the obtained conductivity is quite low compared to conductivity of ideal monolayer graphene, this IV characteristics justify that number of immersion in acetone to dissolve PMMA residues improves the quality of the graphene layer hence improving its conductivity. The overall low conductivity range in Fig. $\mathbf{6}$ may be suggested by the cracks and defects that present in the graphene layer as observed in Fig. 4. Note also the average resistance of $\mathrm{C} 1, \mathrm{C} 2$ and $\mathrm{C} 3$ are $\sim 1.424 \mathrm{E}^{+9}, \sim 2.962 \mathrm{E}^{+5}$ and $1.173 \mathrm{E}^{+5}$ respectively.

\section{Conclusions}

It can be concluded that graphene transfer by using stamping method, specifically using a tool with flat and large surface area (where this work used a wooden block), lead to a large graphene coverage after the transfer process because this method implies a stable pressure during the process. This result is crucial to ensure optimized graphene coverage, which is required for electronic applications. This work has also pointed out the importance of eliminating impurities from the graphene layer in the transfer process, where the impurities are significantly came from PMMA residues. However, this problem can easily be overcomed by cleaning the graphene layer, by immersing the samples repeatedly in solvent, particularly acetone.

\section{References}

1. Novoselov, K. S., Geim, A. K., Morozov, S. V, \& Jiang, D., Science 306, Issue 5696, pp. 666-669, (2004).

2. Yue, W., Jiang, S., Xu, S., Ma, Y., \& Bai, C. Sensors and Actuators B: Chemical, 214, pp. 204-210, (2015).

3. Arefinia, Z., \& Asgari, A. Solar Energy Materials and Solar Cells, 137, pp.146-153, (2015).

4. Li, Z., Zhang, W., Guo, J., Yang, B., \& Yuan, J. Vacuum, 117, 35-39. (2015).

5. Valentini, L. Materials Letters, 148, 204-207. (2015).

6. Ramachandran, R., Saranya, M., Velmurugan, V., Raghupathy, B. P. C., Jeong, S. K., \& Grace, A. N,Applied Energy, 153, 22-31. (2015).

7. Kwon, K.-W., Choi, J.-W., Jung, M. W., You, Y.-H., Chang, R., Seo, J. H., ... Hwang, J.-H. Ceramics International, 41,7, 8360-8366. (2015).

8. Bolotin, K. I., Sikes, K. J., Jiang, Z., Klima, M., Fudenberg, G., Hone, J.,Stormer, H. L.,Solid State Comm. 146,9-10, 351-434. (2008).

9. Lemme, M., \& Echtermeyer, T. Electron Device Letters, 28,4, 282-284. (2007).

10. Lin, Y., Chiu, H., Jenkins, K. A., Member, S., Farmer, D. B., Avouris, P., Abstract, A. DualGate Graphene FETs With f T of $50 \mathrm{GHz}$, 31,1, 2009-2011. (2010).

11. Liao, L., \& Duan, X. Materials Today, 15(78), 328-338. (2012).

12. Reina, A., et al., Nano Letters, 9 (1) ,p, 30-35, (2009).

13. Regan, W..,et al Applied Physics Letters 96,p. 113102,(2010).

14. Ren, Y., et al, Nano, 7, (2012), p.1150001.

15. A. Pirkle, J. Chan, A. Venugopal, D. Hinojos, C. W. Magnuson, S. McDonnell, L. Colombo, E. M. Vogel, R. S. Ruoff, and R. M. Wallace, Appl. Phys. Lett ,99,pp3-5, (2011).

16. J. W. Suk, W. H. Lee, J. Lee, H. Chou, R. D. Piner, Y. Hao, D. Akinwande, and R. S. Ruoff, Nano Lett, 13, 1462 (2013). 